\title{
A Case of an Anomalous Right Coronary Artery Arising from the Left Coronary Cusp
}

\author{
Muhammad Shabbir Rawala ${ }^{1}$, Ahmer A. Longi ${ }^{2}$, Arfeen A. Khan ${ }^{3}$, Syed Imran Rizvi ${ }^{4}$, Syed B. Rizvi ${ }^{5}$ \\ 1. Internal Medicine, Charleston Area Medical Center, Charleston, USA 2. Internal Medicine, Aga Khan University \\ Hospital, Karachi, PAK 3. Internal Medicine, Dr. Ziauddin University and Hospital, Karachi, PAK 4. Cardiac Surgery, \\ Bristol Royal Infirmary, Bristol, GBR 5. Cardiology, Rapides Regional Medical Center, Alexandria, USA
}

Corresponding author: Muhammad Shabbir Rawala, muhammad_rawala@hotmail.com

\begin{abstract}
An anomalous origin of the right coronary artery is usually asymptomatic. It is mostly found incidentally on an invasive diagnostic angiogram. It does lead to an increased risk of sudden cardiac death, especially in younger patients. We present a case of a 41-year-old who had presented to the hospital with complaints of chest pain. The patient was evaluated by cardiology who performed an angiography that identified an anomalous origin of the right coronary artery arising from the left coronary cusp but no evidence of coronary artery disease. Once identified, these anomalous vessels should be corrected surgically, as these conditions increase the risk of sudden cardiac death arrhythmia and ischemic events.
\end{abstract}

Categories: Cardiac/Thoracic/Vascular Surgery, Cardiology, Internal Medicine

Keywords: congenital anomaly, chest pain, coronary artery

\section{Introduction}

Approximately $1 \%$ of patients who undergo cardiac catheterization are reported to have coronary artery anomalies [1]. The incidence of an anomalous right coronary artery originating from the left coronary sinus on coronary angiography is $0.019 \%$ to $0.49 \%$ [1]. Often, patients present with sudden death or myocardial ischemia [2]. Most patients remain asymptomatic and have no ischemic symptoms or findings on resting or stress electrocardiography (ECG); therefore, they are diagnosed only on coronary imaging [3]. We report a case of a 41 -year-old female admitted with complaints of chest pain, who underwent catheterization that detected an anomalous right coronary artery arising from the left coronary cusp.

\section{Case Presentation}

A 41-year-old African American female with a history of hypertension presented to the emergency department (ED) with complaints of chest pain. The patient had experienced intermittent chest pain throughout the night but presented to the ED in the morning after having chest pain along with shortness of breath. On examination, the patient's vitals were stable, and the remaining systemic examination was unremarkable. Cardiology service evaluated the patient; an echocardiogram revealed the left ventricular ejection fraction to be $65 \%$ with no valvular abnormalities. The patient's troponins were negative; however, due to the patient's family history of premature coronary artery disease and the risk factor of hypertension, cardiology decided to evaluate the patient further using invasive angiography (IA). IA identified an anomalous right coronary artery arising from the left coronary cusp (Figures 1-2) and having an anterior inter-arterial course with no evidence of coronary artery disease. The patient was transferred to a tertiary

Received 05/06/2019

Review began 05/10/2019 Published 05/14/2019

\section{(๑) Copyright 2019}

Rawala et al. This is an open access article distributed under the terms of the Creative Commons Attribution License CC-BY 3.0., which permits unrestricted use, distribution, and reproduction in any medium, provided the original author and source are credited. \footnotetext{
care center to be evaluated by cardiothoracic surgery for correction of the anomaly.
} 


\section{Cureus}

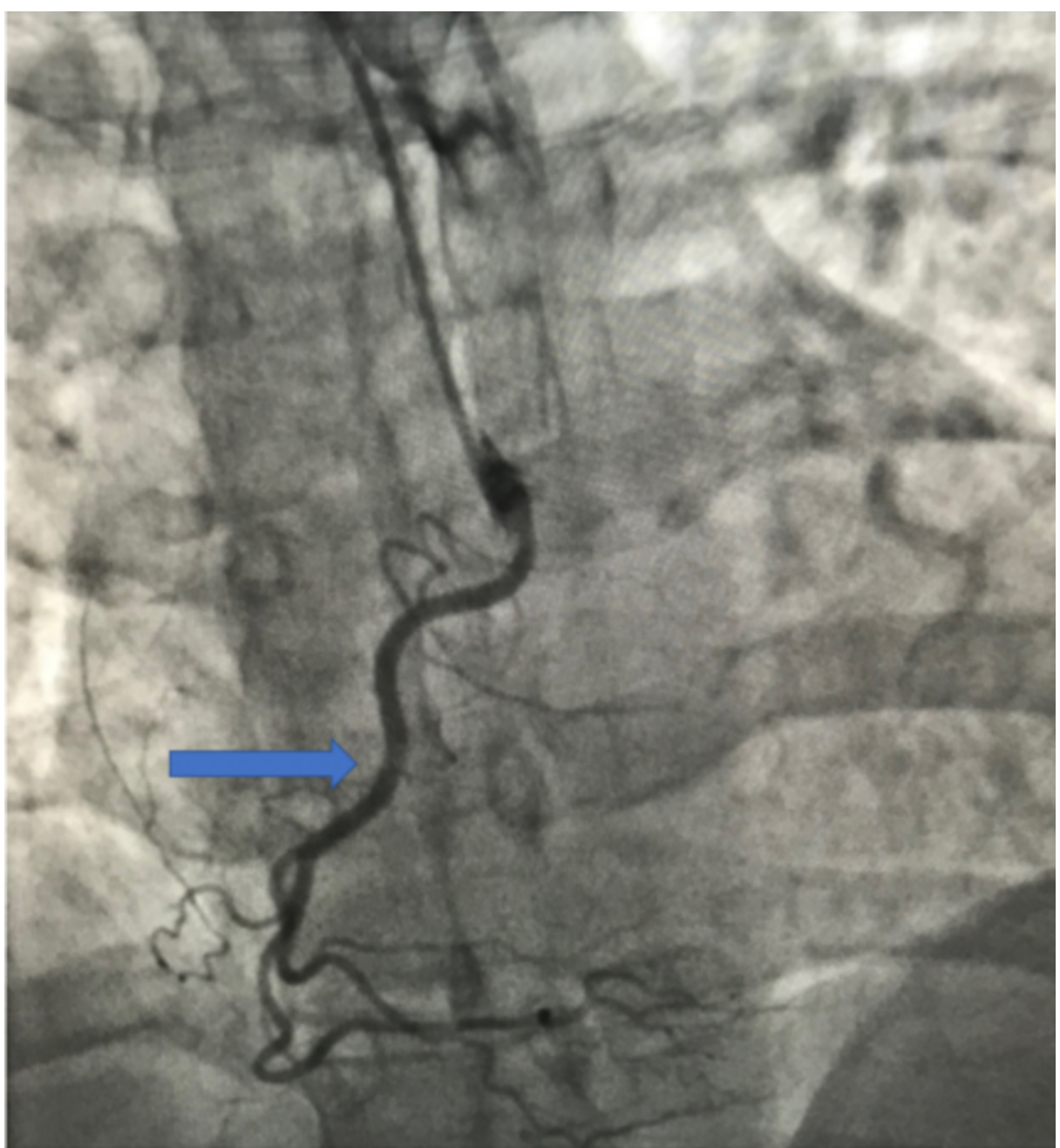

FIGURE 1: Invasive angiogram demonstrating anomalous right coronary artery (arrow) 


\section{Cureus}

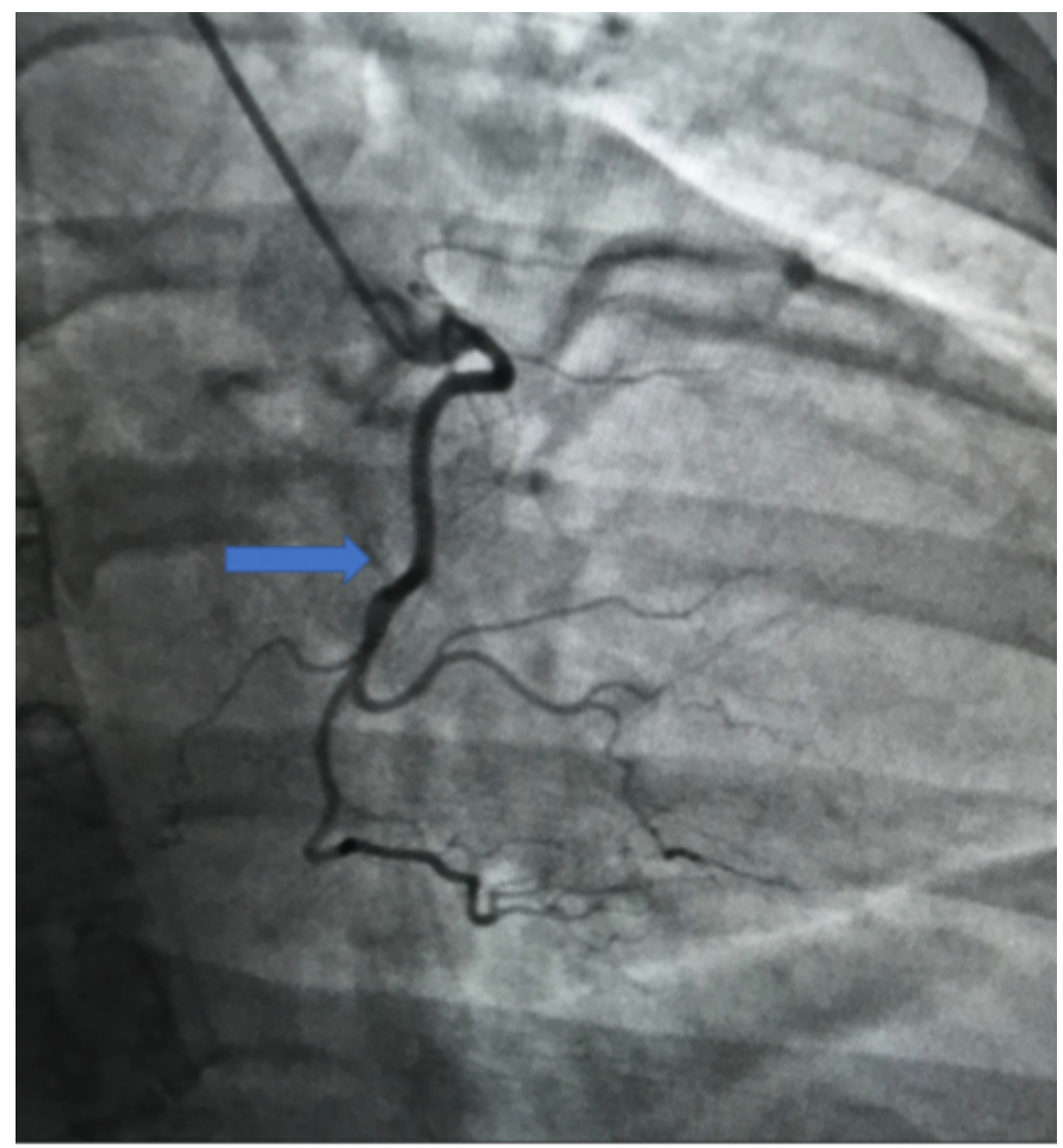

FIGURE 2: Invasive angiogram demonstrating anomalous right coronary artery (arrow)

\section{Discussion}

An anomalous origin of the right coronary artery (RCA) is a rare congenital anomaly that was first described in 1948 by White and Edwards [4]. After carrying out angiography in 126,595 patients, Yamanaka and Hobbs reported the incidence of anomalous origin of the right and left coronary arteries as $136(0.107 \%)$ and 22 $(0.017 \%)$, respectively [5]. The prevalence of an anomalous origin of RCA (ARCA) arising from the left coronary cusp with an inter-arterial course varies between $0.026 \%$ and $0.25 \%$ [6-7]. An ARCA is more common than the anomalous origin of the left coronary artery (ALCA), but the latter is shown to be responsible for up to $85 \%$ of sudden cardiac deaths (SCD) related to the anomalous origins of arteries [8].

Most coronary anomalies are detected incidentally on diagnostic angiography and are clinically insignificant; some, however, have been associated with an increased risk of SCD. It is second only to hypertrophic cardiomyopathy as a leading cause of SCD in young athletes. Mechanical compression of the RCA by the great vessels, which dilate and compress the RCA during periods of increased stroke volume, is the usual explanation for coronary ischemia [9]. Patients usually have no ischemic changes on an electrocardiogram (ECG) at rest; however, multi-detector computed tomography (MDCT) is being used to evaluate the course of anomalous vessels outlying their relative positions to the aorta and the pulmonary artery. It also gives additional information regarding any stenotic lesion in these anomalous vessels [3]. Echocardiography (Echo), magnetic resonance angiography (MRA), MCDT, and cardiac catheterization are all complementary diagnostic tools for evaluating anomalous coronary arteries [10].

While it is agreed that surgical correction is the standard of care for ALCA, when found, the management for right ARCA is more complicated. Treatment options for these patients include observation with medical therapy, percutaneous intervention (stenting), or surgery [11]. Early diagnosis of coronary artery anomalies based on the patients' symptoms can help decrease the incidence of sudden cardiac deaths, fatal arrhythmias, and ischemic events in the high-risk population. 


\section{Conclusions}

Our patient had an anomalous right coronary artery arising from the left coronary cusp, with no evidence of coronary artery disease. It is judicious to understand the anatomy of anomalous vessels beforehand if a cardiac intervention is to be planned, as this may help direct the approach of intervention. Anomalous vessels may lead to sudden cardiac death, arrhythmias, or ischemic events and, therefore, should be corrected when symptomatic.

\section{Additional Information}

\section{Disclosures}

Human subjects: Consent was obtained by all participants in this study. Conflicts of interest: In compliance with the ICMJE uniform disclosure form, all authors declare the following: Payment/services info: All authors have declared that no financial support was received from any organization for the submitted work. Financial relationships: All authors have declared that they have no financial relationships at present or within the previous three years with any organizations that might have an interest in the submitted work. Other relationships: All authors have declared that there are no other relationships or activities that could appear to have influenced the submitted work.

\section{References}

1. Lee BY: Anomalous right coronary artery from the left coronary sinus with an interarterial course: is it really dangerous?. Korean Circ J. 2009, 39:175-179. 10.4070/kcj.2009.39.5.175

2. Sarfaraz ZK, Siddiqi MS, Al-Kindi AH, Alameddine T: Anomalous origin of the right coronary artery from the left coronary sinus: case report. Sultan Qaboos Univ Med J. 2017, 17:352-354. 10.18295/squmj.2017.17.03.017

3. Motomura H, Yokokawa M, Fukunaga H, Nakagaki M, Hasuwa T, Moriuchi H: Anomalous origin of the right coronary artery: first familial cases in Asia. Pediatr Int. 2016, 58:1232-1234. 10.1111/ped.13146

4. White NK, Edwards JE: Anomalies of the coronary arteries; report of four cases . Arch Pathol. 1948, 45:766771.

5. Yamanaka O, Hobbs RE: Coronary artery anomalies in 126,595 patients undergoing coronary arteriography . Cathet Cardiovasc Diagn. 1990, 21:28-40.

6. Alexander RW, Griffith GC: Anomalies of the coronary arteries and their clinical significance . Circulation. 1956, 14:800-805.

7. Kaku B, Shimizu M, Yoshio H, et al.: Clinical features of prognosis of Japanese patients with anomalous origin of the coronary artery. Jpn Circ J. 1996, 60:731-741.

8. Greet B, Quinones A, Srichai M, Bangalore S, Roswell RO: Anomalous right coronary artery and sudden cardiac death. Circ Arrhythm Electrophysiol. 2012, 5:111-112. 10.1161/CIRCEP.112.978635

9. Keren A, Tzivoni D, Stern S: Functional consequences of right coronary artery originating from left sinus of Valsalva. Am J Cardiol. 1983, 51:1241.

10. Moza A, Prashar R, Bawany M: Anomalous origin of right coronary artery associated with hypertrophic obstructive cardiomyopathy. Am J Med Sci. 2011, 342:341-342. 10.1097/MAJ.0b013e318222b607

11. Lilly SM, Schussler JM, Stoler RC: Anomalous origin of the right coronary artery from the left sinus of Valsalva associated with syncope in a young athlete. Proc (Bayl Univ Med Cent). 2011, 24:13-14. 